\title{
CLASSIFICAÇÃO DE ÁRVORES DE EUCALIPTO PARA POSTES EM SISTEMA AGROFLORESTAL ${ }^{1}$
}

\author{
Daniel de Paula Silveira ${ }^{2}$, Helio Garcia Leite ${ }^{3}$, Vicente de Paula Silveira ${ }^{4}$ e Raul Cesar Nogueira Melido ${ }^{4}$
}

\begin{abstract}
RESUMO - Foram construídas tabelas de dupla entrada para quantificação de postes de eucalipto em sistema agroflorestal ( $S A F$ ), seguindo-se as normas Light, ABPM-E86 e CEMIG-ABNT. Além de permitir definição do melhor tipo de poste com base no seu comprimento máximo, ainda com a árvore em pé, as tabelas construídas podem ser empregadas em inventários de postes de eucalipto. Para a construção das tabelas foram utilizados dados de 114 árvores-amostra de Eucalyptus camaldulensis Dehnh. abatidas e cubadas em um sistema agroflorestal, em que os diâmetros e as alturas variavam de 17 a $43 \mathrm{~cm}$ e de 19 a 39 m, respectivamente.
\end{abstract}

Palavras-chave: Postes de eucalipto, Sistema agroflorestal e Afilamento.

\section{CLASSIFICATION OF EUCALYPTUS TREES FOR POLES IN AGROFORESTRY SYSTEM}

\begin{abstract}
Double entered tables for eucalypt poles quantification were constructed in an agroforestry system (AFS), according to the Light, ABPM-E86 and CEMIG ABNT standards. In addition to estimate the best wood pole type based on maximum length, without cutting the tree, the constructed tables can be used in the eucalypt wood poles in forest inventory. To construct the tables, it was used data came from 114 Eucalyptus camaldulensis Dehnh. sample-trees cut and scaled in an agroforestry system, with diameters and heights varying from 17 to $43 \mathrm{~cm}$ and from 19 and $39 \mathrm{~m}$, respectively.
\end{abstract}

Keywords:Agroforestry system, Eucalyptus wood pole and Taper.

\section{INTRODUÇÃO}

O investimento em redes aéreas de distribuição de energia, na maioria das concessionárias brasileiras, é equivalente, ou maior, do que o realizado em subestações e em usinas de geração. De todos os materiais usados nessas redes, os postes, os transformadores, os isoladores, chaves, pára-raios e condutores, os postes representam por volta de $15 \%$ do investimento total (BENTO, 2002).

A demanda anual por postes para distribuição de energia elétrica é considerável. Dados recentes da ANEEL (2008) (Agência Nacional de Energia Elétrica) indicam que, do ano de 1998 até 2007, houve um acréscimo de
24.127,5 km de linhas de transmissão no Sistema Interligado Nacional (SIN). A projeção para o período de 2008 a 2009 é de 7.621,4km. Como consequência, a demanda anual por postes tem aumentado em algumas regiões. Esses postes podem ser de concreto ou de madeira.

Florestas manejadas para produção de postes de madeira são importantes por fixar gás carbônico. Além disso, são renováveis e tem menor custo de produção (cerca de 1.056,0 kcal para fabricação de poste de eucalipto contra 550.000,0 kcal para fabricação do poste de concreto). Os postes de madeira são quase seis vezes mais isolantes elétricos e pesam cerca de $60 \%$ menos do que o concreto, reduzindo os riscos de acidentes e desligamentos por fugas ou descargas elétricas

${ }^{1}$ Recebido em 04.12.2008 e aceito para publicação em 20.04.2011.

${ }^{2}$ Graduação em andamento em Engenharia Florestal pela Universidade Federal de Viçosa, UFV, Brasil. E-mail: <daniel.silveira@ufv.br>.

${ }^{3}$ Departamento de Engenharia Florestal, Universidade Federal de Viçosa, UFV, Brasil. E-mail: <hgleite@gmail.com>.

${ }^{4}$ Siderurgica Barra Mansa S/A, SBM, Brasil. 
e, também, o custo de transporte. Em razão de sua elevada elasticidade, os postes de madeira também apresentam maior resistência aos choques mecânicos, quando comparados a outros tipos de postes (REMADE, 2003)

O preço de um poste de madeira de $11 \mathrm{~m} \mathrm{(400} \mathrm{kg)}$ é cerca de $60 \%$ do valor de um poste de concreto de mesmo tamanho, e o custo de implantação de um poste de madeira é em torno de $55 \%$ do valor do poste de concreto (ABPM, 2005). Esse e outros tipos de postes podem ser obtidos em povoamentos de eucalipto, em monocultura ou sob sistemas agroflorestais.

A existência de diferentes tipos de postes é consequência da existência de diferentes tabelas, como ABNT e Light. Tabelas de postes de eucalipto foram apresentadas em 1986 no Sexto Congresso Florestal Brasileiro e publicadas por Campos et al. (1991). As tabelas foram construídas por esses autores, com dados de Eucalyptus grandis de povoamentos localizados no Sul da Bahia, seguiram duas normas de classificação (ABNT e Ligth). Para construção das tabelas, eles utilizaram o modelo de Demaerschalk (1972).

MacDicken e Vergara (1990) e Young (1994) denominaram sistemas agroflorestais (SAFs), genericamente, os sistemas produtivos que incluem árvores em consórcio e, ou, associação com culturas agrícolas e, ou, com criação de animais (VIEIRA et al., 2003).

Considerando que árvores de eucalipto oriundas de sistemas agroflorestais (SAF) têm sido utilizadas para postes no Estado de Minas Gerais e a existência de uma terceira tabela de classificação de postes, da Central de Energia Elétrica de Minas Gerais - CEMIG, foi conduzido este estudo, com o seguinte objetivo: construir e avaliar a precisão de tabelas de classificação de postes de eucalipto em SAF, em função da altura da árvore e do diâmetro a 1,30 m, de acordo com três normas diferentes.

\section{MATERIAL E MÉTODOS}

\section{1. Área de estudo}

Os dados para este estudo foram obtidos em talhões de Eucalyptus camaldulensis, situados na Fazenda Riacho, pertencente à empresa Votorantim Siderurgia, Unidade Florestal. A fazenda está localizada no Município de Paracatu, MG, com latitude é 17³6’09”' Sul e longitude 4642’02”' Oeste de Greenwich, numa altitude de $550 \mathrm{~m}$. Segundo a classificação climática de Köppen, a região é do tipo Aw, clima tropical úmido de savana, com inverno seco e verão chuvoso (ANTUNES, 1986). A temperatura média anual é de $24^{\circ} \mathrm{C}$ e precipitação média anual de $1.250 \mathrm{~mm}$.

\subsection{Dados e análises}

Cento e quatorze árvores-amostra, com diâmetro a 1,3 m de altura (dap) variando de 19 a $43 \mathrm{~cm}$ e altura total entre 19 e $39 \mathrm{~m}$, foram selecionadas para abate e cubagem. Os diâmetros com e sem casca foram medidos em seções de 1,0 m até um diâmetro comercial de cerca de 4 cm.

As árvores foram classificadas em classes de 2,0 cm de dap e agrupadas casualmente em dois grupos, sendo um de 84 árvores (Tabela 1), utilizado no ajustamento do modelo de afilamento e modelo de altura, e outro de 30 árvores, empregado na validação das equações estimadas.

Para garantir a interpretação dos objetivos e resultados deste estudo, julgou-se necessário reproduzir as características dos postes por classe ou tipo, de acordo com os padrões Light, ABPM-E86 e CEMIG (Tabelas 2, 3 e 4).

O modelo de regressão utilizado para estimar a altura total das árvores em função do diâmetro, a 1,3 m de altura (dap), foi o modelo logístico, sendo:

$$
H=\frac{\beta_{0}}{1+\beta_{1} e^{-\beta_{2} d a p}}+\varepsilon
$$

em que:

$H=$ altura total da árvore, em m;

dap = diâmetro com casca na altura 1,30 m, em cm; e $\varepsilon=$ erro aleatório.

O modelo de regressão utilizado para representação do perfil do tronco das árvores foi proposto por Garay (1979), sendo:

$$
d=\operatorname{dap} \beta_{0}\left(1+\beta_{1} \ln \left(1-\beta_{2} h^{\beta_{3}} H^{-\beta_{3}}\right)\right)+\varepsilon
$$

em que:

$d$ = diâmetro sem casca, em cm, à altura determinada do tronco;

dap = diâmetro com casca na altura 1,30 m, em cm; $h$ = distância do nível do chão até o diâmetro d, em m;

$H=$ altura total da árvore, em m; e

$\varepsilon=$ erro aleatório. 
Tabela 1 - Distribuição de frequência das árvores-amostra utilizadas no ajustamento da equação de afilamento e altura total. Table 1 -Distribution of frequency of the tree-samples used in the adjustment of taper and total height equation.

\begin{tabular}{|c|c|c|c|c|c|c|c|c|c|c|c|c|c|c|c|}
\hline \multirow{2}{*}{$\begin{array}{l}\text { Classes de } \\
\text { altura(m) }\end{array}$} & \multicolumn{14}{|c|}{ Classes de diâmetro $(\mathrm{cm})$} & \multirow[t]{2}{*}{ Total } \\
\hline & 17 & 19 & 21 & 23 & 25 & 27 & 29 & 31 & 33 & 35 & 37 & 39 & 41 & 43 & \\
\hline 19 & 1 & 3 & 2 & & & & & & & & & & & & 6 \\
\hline 21 & 2 & 1 & & 2 & & & & & & & & & & & 5 \\
\hline 23 & 3 & 2 & 1 & & & & & & & & & & & & 6 \\
\hline 25 & & & 3 & 3 & 1 & & & & & & & & & & 7 \\
\hline 27 & & & & 1 & 3 & 2 & & 1 & 1 & & & & & & 8 \\
\hline 29 & & & & & 2 & 3 & & 1 & & & & & & & 6 \\
\hline 31 & & & & & & 1 & 4 & & 2 & & 1 & 1 & 1 & & 10 \\
\hline 33 & & & & & & & 2 & 1 & 1 & 2 & 1 & 2 & 1 & 3 & 13 \\
\hline 35 & & & & & & & & 2 & 1 & 2 & 2 & 1 & 2 & & 10 \\
\hline 37 & & & & & & & & 1 & 1 & 2 & 2 & 2 & 2 & 2 & 12 \\
\hline 39 & & & & & & & & & & & & & & 1 & 1 \\
\hline Total & 6 & 6 & 6 & 6 & 6 & 6 & 6 & 6 & 6 & 6 & 6 & 6 & 6 & 6 & 84 \\
\hline
\end{tabular}

Tabela 2 - Características de postes de eucalipto segundo o padrão de classificação Light. Table 2 - Eucalyptus pole characteristics according to Light classification standard.

\begin{tabular}{|c|c|c|c|c|c|c|c|c|}
\hline \multirow{2}{*}{$\begin{array}{c}\text { Comprimento } \\
\text { total }\end{array}$} & \multirow{2}{*}{$\begin{array}{l}\text { Classe } \\
\text { ou tipo }\end{array}$} & \multirow{2}{*}{$\begin{array}{c}\text { Carga } \\
\text { nominal } \\
\text { a } 45 \mathrm{~cm} \\
\text { do topo }\end{array}$} & \multirow{2}{*}{$\begin{array}{l}\text { Distância } \\
\text { da linha de } \\
\text { afloramento } \\
\text { da base }\end{array}$} & \multirow{2}{*}{$\begin{array}{l}\text { Comprimento } \\
\text { útil }\end{array}$} & \multicolumn{2}{|c|}{ Circunferências } & \multicolumn{2}{|c|}{ Diâmetro } \\
\hline & & & & & $\begin{array}{c}\text { Mínima a } \\
1,8 \mathrm{~m} \\
\text { da base }\end{array}$ & $\begin{array}{l}\text { Mínima } \\
\text { no topo } \\
\text { do poste }\end{array}$ & $\begin{array}{l}\text { Mínimo } \\
\text { a } 1,8 \mathrm{~m} \\
\text { da base }\end{array}$ & $\begin{array}{l}\text { Mínimo } \\
\text { no topo } \\
\text { do poste }\end{array}$ \\
\hline $\mathrm{m}$ & & $\mathrm{kg}$ & $\longrightarrow \mathrm{m}$ & 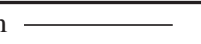 & $\longrightarrow$ & $\mathrm{cm}$ & 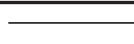 & $\bar{Z}$ \\
\hline 7 & Leve & 230 & 1,4 & 5,6 & 54 & 43 & 17,2 & 13,7 \\
\hline 8 & Leve & 230 & 1,4 & 6,6 & 58 & 44 & 18,5 & 14 \\
\hline 9 & Leve & 230 & 1,7 & 7,3 & 61 & 45 & 19,4 & 14,3 \\
\hline 9 & Médio & 360 & 1,7 & 7,3 & 70 & 54 & 22 & 17,2 \\
\hline 9 & Pesado & 590 & 1,7 & 7,3 & 83 & 67 & 26,4 & 21,3 \\
\hline 10 & Leve & 230 & 1,8 & 8,2 & 64 & 46 & 20,4 & 14 \\
\hline 10 & Médio & 360 & 1,8 & 8,2 & 74 & 56 & 23,6 & 17,8 \\
\hline 10 & Pesado & 590 & 1,8 & 8,2 & 86 & 68 & 27,4 & 24,6 \\
\hline 11 & Leve & 230 & 1,8 & 9,2 & 67 & 47 & 21,3 & 15 \\
\hline 11 & Médio & 360 & 1,8 & 9,2 & 77 & 57 & 24,5 & 15,1 \\
\hline 11 & Pesado & 590 & 1,8 & 9,2 & 90 & 70 & 28,6 & 22,3 \\
\hline 12 & Leve & 230 & 1,9 & 10,1 & 70 & 48 & 22,3 & 15,3 \\
\hline 12 & Médio & 360 & 1,9 & 10,1 & 80 & 58 & 25,4 & 18,5 \\
\hline 12 & Pesado & 590 & 1,9 & 10,1 & 93 & 71 & 29,6 & 22,6 \\
\hline 13 & Médio & 270 & 1,9 & 11,1 & 76 & 51 & 24,2 & 16,2 \\
\hline 13 & Pesado & 450 & 1,9 & 11,1 & 89 & 64 & 28,3 & 20,4 \\
\hline 14 & Médio & 270 & 2 & 12 & 79 & 52 & 25,2 & 16,5 \\
\hline 14 & Pesado & 450 & 2 & 12 & 92 & 65 & 29,3 & 20,9 \\
\hline 15 & Médio & 270 & 2 & 13 & 81 & 52 & 25,8 & 16,5 \\
\hline 15 & Pesado & 450 & 2 & 13 & 93 & 64 & 29,6 & 20,4 \\
\hline 16 & Médio & 270 & 2 & 14 & 84 & 53 & 26,7 & 16,9 \\
\hline 16 & Pesado & 450 & 2 & 14 & 98 & 67 & 31,2 & 21,3 \\
\hline 17 & Médio & 270 & 2 & 15 & 88 & 55 & 28 & 17,5 \\
\hline 17 & Pesado & 450 & 2 & 15 & 102 & 69 & 32,4 & 22 \\
\hline 18 & Médio & 270 & 2 & 16 & 90 & 55 & 28,6 & 17,5 \\
\hline 18 & Pesado & 450 & 2 & 16 & 104 & 69 & 33,1 & 22 \\
\hline
\end{tabular}


Tabela 3 - Características de postes de eucalipto segundo o padrão de classificação ABPM-E86.

Table 3 - Eucalyptus pole characteristics according to ABPM-E86 classification standard.

\begin{tabular}{|c|c|c|c|c|c|c|c|c|}
\hline \multirow{2}{*}{$\begin{array}{c}\text { Comprimento } \\
\text { total }\end{array}$} & \multirow{2}{*}{$\begin{array}{l}\text { Classe } \\
\text { ou tipo }\end{array}$} & \multirow{2}{*}{$\begin{array}{c}\text { Carga } \\
\text { nominal } \\
\text { a } 45 \mathrm{~cm} \\
\text { do topo }\end{array}$} & \multirow{2}{*}{$\begin{array}{c}\text { Distância } \\
\text { da linha de } \\
\text { afloramento } \\
\text { da base }\end{array}$} & \multirow{2}{*}{$\begin{array}{c}\text { Comprimento } \\
\text { útil }\end{array}$} & \multicolumn{2}{|c|}{ Circunferências } & \multicolumn{2}{|c|}{ Diâmetro } \\
\hline & & & & & $\begin{array}{c}\text { Mínima a } \\
1,8 \mathrm{~m} \\
\text { da base }\end{array}$ & $\begin{array}{l}\text { Mínima } \\
\text { no topo } \\
\text { do poste }\end{array}$ & $\begin{array}{l}\text { Mínimo } \\
\text { a } 1,8 \mathrm{~m} \\
\text { da base }\end{array}$ & $\begin{array}{l}\text { Mínimo } \\
\text { no topo } \\
\text { do poste }\end{array}$ \\
\hline $\mathrm{m}$ & & $\mathrm{kg}$ & $\longrightarrow$ & 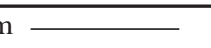 & & $c$ & 11 & 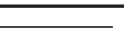 \\
\hline 7 & E.Leve & 150 & 1,3 & 5,4 & 48 & $28-37$ & 14,2 & 8,9 \\
\hline 7 & Leve & 250 & 1,3 & 5,4 & 53 & $36-45$ & 17 & 11,5 \\
\hline 7 & Médio & 400 & 1,3 & 5,4 & 62 & $45-54$ & 19,8 & 14,5 \\
\hline 7 & Pesado & 600 & 1,3 & 5,4 & 71 & $54-63$ & 22,7 & 17,3 \\
\hline 8 & E.Leve & 150 & 1,4 & 6,3 & 48 & $28-39$ & 15,2 & 8,9 \\
\hline 8 & Leve & 250 & 1,4 & 6,3 & 56 & $36-47$ & 17,8 & 11,5 \\
\hline 8 & Médio & 400 & 1,4 & 6,3 & 66 & $46-56$ & 20,9 & 14,6 \\
\hline 8 & Pesado & 600 & 1,4 & 6,3 & 75 & $55-66$ & 23,9 & 17,6 \\
\hline 9 & E.Leve & 150 & 1,5 & 7,2 & 51 & $28-40$ & 16,1 & 8,9 \\
\hline 9 & Leve & 250 & 1,5 & 7,2 & 59 & $36-48$ & 18,7 & 11,5 \\
\hline 9 & Médio & 400 & 1,5 & 7,2 & 68 & $46-57$ & 21,8 & 14,6 \\
\hline 9 & Pesado & 600 & 1,5 & 7,2 & 78 & 56-69 & 24,9 & 17,7 \\
\hline 10 & E.Leve & 150 & 1,6 & 8,1 & 54 & $28-42$ & 17 & 8,9 \\
\hline 10 & Leve & 250 & 1,6 & 8,1 & 62 & $36-50$ & 19,6 & 11,5 \\
\hline 10 & Médio & 400 & 1,6 & 8,1 & 71 & $46-59$ & 22,7 & 14,5 \\
\hline 10 & Pesado & 600 & 1,6 & 8,1 & 81 & $56-70$ & 25,9 & 17,7 \\
\hline 11 & E.Leve & 150 & 1,7 & 9 & 56 & $28-43$ & 17,9 & 8,9 \\
\hline 11 & Leve & 250 & 1,7 & 9 & 64 & $36-51$ & 20,5 & 11,5 \\
\hline 11 & Médio & 400 & 1,7 & 9 & 74 & $46-61$ & 23,6 & 14,6 \\
\hline 11 & Pesado & 600 & 1,7 & 9 & 84 & $56-71$ & 26,8 & 17,8 \\
\hline 12 & E.Leve & 250 & 1,8 & 9,9 & 67 & $36-53$ & 21,4 & 11,5 \\
\hline 12 & Leve & 400 & 1,8 & 9,9 & 77 & $46-62$ & 24,5 & 14,6 \\
\hline 12 & Médio & 600 & 1,8 & 9,9 & 87 & $56-73$ & 27,8 & 17,9 \\
\hline 12 & Pesado & 800 & 1,8 & 9,9 & 96 & $65-81$ & 30,5 & 20,6 \\
\hline 13 & Leve & 250 & 1,9 & 10,8 & 70 & $36-54$ & 22,3 & 11,5 \\
\hline 13 & Médio & 400 & 1,9 & 10,8 & 80 & $46-64$ & 25,4 & 14,6 \\
\hline 13 & Pesado & 600 & 1,9 & 10,8 & 90 & 56-74 & 28,7 & 17,9 \\
\hline 13 & E.Pesado & 800 & 1,9 & 10,8 & 99 & $65-83$ & 31,4 & 20,6 \\
\hline 14 & Leve & 250 & 2 & 11,7 & 73 & $36-56$ & 23,2 & 11,5 \\
\hline 14 & Médio & 400 & 2 & 11,7 & 83 & $46-55$ & 26,3 & 14,6 \\
\hline 14 & Pesado & 600 & 2 & 11,7 & 93 & $56-76$ & 29,6 & 17,9 \\
\hline 14 & E.Pesado & 800 & 2 & 11,7 & 101 & $65-84$ & 32,3 & 20,6 \\
\hline 15 & Médio & 400 & 2,1 & 12,6 & 85 & $46-67$ & 27,2 & 14,6 \\
\hline 15 & Pesado & 600 & 2,1 & 12,6 & 96 & $56-77$ & 30,5 & 17,9 \\
\hline 15 & E.Pesado & 800 & 2,1 & 12,6 & 104 & $65-86$ & 33,2 & 20,6 \\
\hline 16 & Médio & 400 & 2,2 & 13,5 & 88 & $46-68$ & 28,1 & 14,6 \\
\hline 16 & Pesado & 600 & 2,2 & 13,5 & 99 & 56-79 & 31,4 & 17,9 \\
\hline 16 & E.Pesado & 800 & 2,2 & 13,5 & 107 & $65-87$ & 34,1 & 20,6 \\
\hline 17 & Médio & 400 & 2,3 & 14,4 & 91 & $46-70$ & 29 & 14,6 \\
\hline 17 & Pesado & 600 & 2,3 & 14,4 & 101 & $56-80$ & 32,3 & 17,9 \\
\hline 17 & E.Pesado & 800 & 2,3 & 14,4 & 110 & 65-89 & 35 & 20,6 \\
\hline 18 & Médio & 400 & 2,4 & 15,3 & 94 & $46-71$ & 29,9 & 14,6 \\
\hline 18 & Pesado & 600 & 2,4 & 15,3 & 104 & $56-82$ & 33,2 & 17,9 \\
\hline 18 & E.Pesado & 800 & 2,4 & 15,2 & 113 & $65-90$ & 35,9 & 20,6 \\
\hline 19 & Médio & 400 & 2,5 & 16,2 & 97 & $46-73$ & 30,8 & 14,6 \\
\hline 19 & Pesado & 600 & 2,5 & 16,2 & 107 & $56-83$ & 34,1 & 17,9 \\
\hline 19 & E.Pesado & 800 & 2,5 & 16,2 & 116 & $65-92$ & 36,8 & 20,6 \\
\hline 20 & Médio & 400 & 2,6 & 17,1 & 100 & $46-74$ & 31,7 & 14,6 \\
\hline 20 & Pesado & 600 & 2,6 & 17,1 & 110 & 56-85 & 35 & 17,9 \\
\hline 20 & E.Pesado & 800 & 2,6 & 17,1 & 18 & $65-93$ & 37,7 & 20,6 \\
\hline
\end{tabular}

Revista Árvore, Viçosa-MG, v.35, n.4., p.875-882, 2011 
Tabela 4 - Características de postes de eucalipto segundo o padrão de classificação CEMIG. Table 4 - Eucalyptus pole characteristics according to CEMIG classification standard.

\begin{tabular}{|c|c|c|c|c|c|c|c|c|c|c|c|}
\hline \multirow{3}{*}{$\begin{array}{c}\text { Comprimento } \\
\text { total }\end{array}$} & \multirow{3}{*}{$\begin{array}{l}\text { Classe } \\
\text { ou tipo }\end{array}$} & \multirow{3}{*}{$\begin{array}{c}\text { Resist. } \\
\text { nominal } \\
\text { Rn (daN) }\end{array}$} & \multicolumn{5}{|c|}{ Ponto de engastamento } & \multicolumn{4}{|c|}{ Ponta-cm } \\
\hline & & & \multirow{2}{*}{$\begin{array}{l}\text { Altura } \\
\text { do pé }\end{array}$} & \multicolumn{2}{|c|}{ Circunferência } & \multicolumn{2}{|c|}{ Diâmetro } & \multicolumn{2}{|c|}{ Circunferência } & \multicolumn{2}{|c|}{ Diâmetro } \\
\hline & & & & Mín. & Média & Mín. & Média & Mín. & Máxima & Mín. & Máxima \\
\hline $\mathrm{m}$ & & $\mathrm{kg}$ & $\mathrm{m}$ & & & & 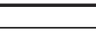 & $\mathrm{m}$ & & & \\
\hline 9 & Leve & 150 & 1,50 & 58 & 58 & 18,5 & 18,5 & 35 & 51 & 11,1 & 16,2 \\
\hline \multirow[t]{3}{*}{10} & Leve & 150 & 1,60 & 62 & 62 & 19,7 & 19,7 & 36 & 52 & 11,5 & 16,6 \\
\hline & Médio & 300 & & 73 & 73 & 23,2 & 23,2 & 46 & 62 & 14,6 & 19,7 \\
\hline & Pesado & 600 & & 82 & 82 & 26,1 & 26,1 & 56 & 72 & 17,8 & 22,9 \\
\hline \multirow[t]{2}{*}{11} & Médio & 300 & 1,70 & 77 & 77 & 24,5 & 24,5 & 48 & 64 & 15,3 & 20,4 \\
\hline & Pesado & 600 & & 85 & 85 & 27,1 & 27,1 & 56 & 72 & 17,8 & 22,9 \\
\hline \multirow[t]{2}{*}{12} & Médio & 300 & 1,80 & 81 & 81 & 25,8 & 25,8 & 49 & 65 & 15,6 & 20,7 \\
\hline & Pesado & 600 & & 89 & 89 & 28,3 & 28,3 & 57 & 73 & 18,1 & 23,2 \\
\hline \multirow[t]{2}{*}{13} & Médio & 300 & 1,90 & 92 & 92 & 29,3 & 29,3 & 40 & 56 & 12,7 & 17,8 \\
\hline & Pesado & 600 & & 100 & 100 & 31,8 & 31,8 & 48 & 64 & 15,3 & 20,4 \\
\hline \multirow[t]{2}{*}{14} & Médio & 300 & 2,00 & 97 & 97 & 30,9 & 30,9 & 40 & 56 & 12,7 & 17,8 \\
\hline & Pesado & 600 & & 105 & 105 & 33,4 & 33,4 & 49 & 65 & 15,6 & 20,7 \\
\hline \multirow[t]{2}{*}{15} & Médio & 300 & 2,10 & 101 & 101 & 32,1 & 32,1 & 41 & 57 & 13,1 & 18,1 \\
\hline & Pesado & 600 & & 110 & 110 & 35,0 & 35,0 & 50 & 65 & 15,9 & 20,7 \\
\hline 16 & Pesado & 600 & 2,20 & 115 & 115 & 36,6 & 36,6 & 50 & 66 & 15,9 & 21,0 \\
\hline 17 & Pesado & 600 & 2,30 & 118 & 118 & 37,6 & 37,6 & 51 & 66 & 16,2 & 21,0 \\
\hline 18 & Pesado & 600 & 2,40 & 124 & 124 & 39,5 & 39,5 & 51 & 67 & 16,2 & 21,3 \\
\hline 19 & Pesado & 600 & 2,50 & 129 & 129 & 41,1 & 41,1 & 52 & 68 & 16,6 & 21,6 \\
\hline 20 & Pesado & 600 & 2,60 & 134 & 134 & 42,7 & 42,7 & 52 & 68 & 16,6 & 21,6 \\
\hline
\end{tabular}

A decisão de empregar esse modelo foi decorrente de resultados obtidos em estudos anteriores, entre eles Leite et al. (2006), que avaliaram a qualidade do ajustamento desse modelo com base na correlação entre valores observados e valores estimados do diâmetro comercial (diâmetro ao longo do fuste).

A validação das regressões estimadas para determinar altura total das árvores e afilamento do fuste foi efetuada usando-se os dados das 30 árvores sorteadas. Em ambos os casos, a análise de resíduos foi realizada para verificar a exatidão das estimativas. Para avaliar o modelo 1, foram também estimados o coeficiente de correlação $\left(\gamma_{\hat{H} H}\right)$, o desvio-padrão amostral ( $\left.\mathrm{s}_{\mathrm{xy}}\right)$ e o coeficiente de variação (CV). Para avaliar a eficiência do procedimento de classificação de postes, foram estimados para a equação de taper o coeficiente de correlação $\left(\gamma_{\hat{d} d}\right)$, a média das diferenças absolutas (MDA), a média das diferenças percentuais (MDP), o erro de tendência (bias) e raiz quadrada do erro médio (RQEM), a 4 e 16 m da altura comercial, utilizando dados da amostra independente de 30 árvores. Essas estatísticas foram interpretadas em termos relativos, em comparação com estudos semelhantes e foram estimadas por:

$$
\begin{gathered}
\text { Bias }=n^{-1}\left(\sum_{i=1}^{n}\right) d_{i}-\sum_{i=1}^{n} \hat{d}_{i} \\
R Q E M=100 d^{-1} \sqrt{n^{-1} \sum_{i=1}^{n}\left(d_{1}-\hat{d}_{i}\right)^{2}} \\
M D A=\left(n^{-1} \sum_{i=1}^{n} \mid d_{i}-\hat{d}_{i}\right) \mathrm{e} \\
M D P=n^{-1} \sum_{i=1}^{n} 100\left(\frac{d_{i} \hat{d}_{i}}{d_{i}}\right)
\end{gathered}
$$

em que $d_{i}, \hat{d}_{i}, \bar{d}=$ são, respectivamente, diâmetro observado, diâmetro estimado pelo modelo e média dos diâmetros observados.

A composição dos tipos de postes, cujas dimensões foram estimadas a partir da equação oriunda do ajuste do modelo 2, foi baseada no aproveitamento do máximo comprimento do fuste. Essa decisão de se basear no máximo comprimento foi para maximizar o aproveitamento da madeira da árvore. Para efeito dessa classificação de cada poste, foram construídas tabelas que proporcionaram o tipo de poste de maior comprimento, segundo as dimensões contidas nos três padrões de classificação.

Revista Árvore, Viçosa-MG, v.35, n.4, p.875-882, 2011 


\section{RESULTADOS E DISCUSSÃO}

As equações de afilamento e altura total ajustadas foram, respectivamente:

$$
\begin{aligned}
& \hat{d}=\operatorname{dap}(1,07830)\left(1+0,491766 \operatorname{Ln}(1-0,871957) h^{0,400224} H^{-0400224}\right) ; \\
& r_{\hat{d} d}=0,981 \\
& \hat{H}=\frac{37,003626}{1+8,336552 e^{-0,126396 d a p}} ; \\
& \operatorname{com} r_{\hat{H} H}=0,897, s_{y x}=2,575 \mathrm{~m}, \mathrm{cv}=8,627 \%
\end{aligned}
$$

Os tipos de postes de maior altura obtidos para cada combinação de dap e $H$ foram relacionados na Tabela 5.

A comparação dos resultados da Tabela 5 indica que as normas adotadas pela ABPM proporcionaram maior racionalidade na utilização do tronco, pois essas normas classificam também os postes de menores dimensões. Por exemplo, árvores de diâmetro 19,0 cm e altura total de 19,0 m resultam postes do tipo EL9, pela norma ABPM, e nenhum tipo, pelas normas Light e CEMIG.

As estimativas de MDA, MDP, bias e RQEM nas alturas comerciais de 4 e $16 \mathrm{~m}$ foram: $\mathrm{MDA}_{4}=1,1502$, $\mathrm{MDA}_{16}=1,2831, \mathrm{MDP}_{4}=1,1406, \mathrm{MDP}_{16}=-0,8764$, bias $_{4}$ $=-0,2699$, bias $_{16}=0,2557$, RQEM$_{4}=7,4451$ e RQEM $_{16}$ $=13,0837$.

Comparando algumas dessas estimativas com resultados encontrados por Lima (1986), que analisou funções de taper em Pinus elliottii Engelm., verificou-se concordância entre as estimativas de ambos os trabalhos, indicando que a equação 3 estima diâmetros a diferentes alturas da árvore com precisão.

A equação 4 apresentou ajuste satisfatório, tendo como base as estimativas calculadas $\left(r_{\hat{H} H}, s_{y x}, C V\right)$ e a análise de resíduo da amostra independente de 30 árvores.

Os gráficos de resíduo, em percentagem, para as equações 3 e 4 utilizando dados da amostra independente de 30 árvores são apresentados, respectivamente, nas Figuras 1 e 2.

Somente um tipo de poste foi determinado para cada árvore, em vista da opção de máximo comprimento adotada, que implica obter o tipo de poste de maior comprimento possível em cada árvore. Contudo, a metodologia apresentada permite estimar postes a partir de outro critério, o que poderia até resultar em mais de um poste por árvore.
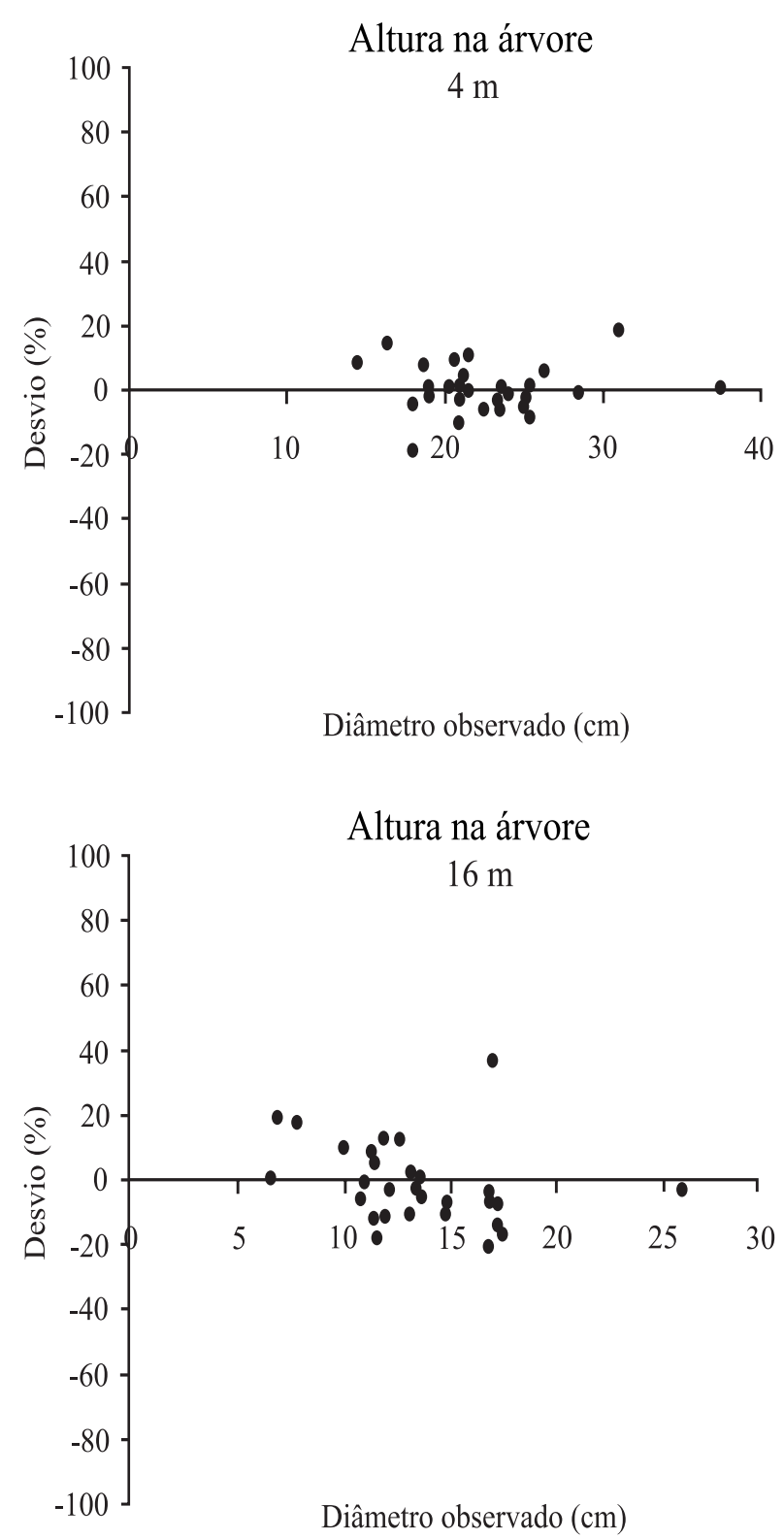

Figura 1 - Distribuição dos desvios percentuais entre os valores observados e recuperados pela equação 3 utilizando os dados das 30 árvores-amostra.

Figure 1 -Distribution of percentage deviations between values observed and recovered by equation 3 using data from 30 sample-trees. 
Tabela 5 - Tipos de postes obtidos em árvores de eucalipto, em sistema agroflorestal, segundo o padrão ABPM, Light e CEMIG, e critério de classificação do máximo comprimento ${ }^{6}$.

Table 5 - Types of poles obtained from eucalyptus trees, in agroforestry system, according to standard ABPM, Light and CEMIG, and criterion of maximum length ${ }^{6}$.

\begin{tabular}{|c|c|c|c|c|c|c|c|c|c|c|c|c|c|c|c|c|c|}
\hline \multirow[t]{2}{*}{ Classificação } & \multicolumn{2}{|c|}{$\begin{array}{l}\text { DAP } \\
(\mathrm{cm})\end{array}$} & \multicolumn{15}{|c|}{ Altura total (m) } \\
\hline & & 11 & 13 & 15 & 17 & 19 & 21 & 23 & 25 & 27 & 29 & 31 & 33 & 35 & 37 & 39 & 41 \\
\hline \multirow{15}{*}{ АВРМ } & 17 & & & & EL7 & EL7 & EL7 & EL7 & EL7 & EL7 & EL8 & EL8 & EL8 & EL8 & EL8 & EL8 & EL8 \\
\hline & 19 & & EL7 & EL8 & EL8 & EL9 & EL9 & EL9 & EL9 & EL9 & EL10 & EL10 & EL10 & EL10 & EL10 & EL10 & EL10 \\
\hline & 21 & & EL7 & EL8 & EL10 & EL11 & EL11 & EL11 & EL11 & EL11 & EL11 & EL11 & EL11 & EL11 & EL11 & EL11 & EL11 \\
\hline & 23 & EL7 & EL8 & EL9 & EL10 & EL11 & EL11 & EL11 & EL11 & L11 & L11 & L11 & L11 & L11 & L11 & L11 & L11 \\
\hline & 25 & EL7 & EL8 & EL10 & EL11 & EL11 & L11 & EL12 & EL12 & L13 & L13 & L13 & L13 & L13 & L13 & L13 & L13 \\
\hline & 27 & EL7 & EL9 & EL10 & EL11 & L11 & EL12 & L13 & L14 & L14 & L14 & L14 & L14 & L14 & L14 & L14 & L14 \\
\hline & 29 & EL7 & EL9 & EL10 & EL11 & L11 & L13 & L14 & L14 & M13 & M13 & M13 & M14 & M14 & M14 & M14 & M14 \\
\hline & 31 & EL8 & EL9 & EL11 & L11 & EL12 & L13 & L14 & M13 & M14 & M15 & M15 & M16 & M16 & M16 & M16 & M16 \\
\hline & 33 & EL8 & EL9 & EL11 & L11 & EL12 & L14 & M13 & M14 & M15 & M16 & M17 & M18 & M18 & M18 & M18 & M18 \\
\hline & 35 & EL8 & EL10 & EL11 & L11 & L13 & L14 & M13 & M15 & M16 & M17 & M18 & M19 & M20 & M20 & M20 & M20 \\
\hline & 37 & EL8 & EL10 & EL11 & EL12 & L13 & L14 & M14 & M15 & M16 & M18 & M19 & M20 & M20 & M20 & M20 & M20 \\
\hline & 39 & L8 & EL10 & L11 & EL12 & L14 & L14 & M15 & M16 & M17 & M18 & M20 & M20 & M20 & P20 & P20 & P20 \\
\hline & 41 & L8 & EL10 & L11 & EL12 & L14 & M14 & M15 & M16 & M18 & M19 & M20 & M20 & P20 & P20 & P20 & EP20 \\
\hline & 43 & EL9 & EL10 & L11 & L13 & L14 & M14 & M15 & M17 & M18 & M20 & M20 & M20 & P20 & P20 & EP20 & EP20 \\
\hline & 45 & EL9 & L10 & L11 & L13 & L14 & M14 & M16 & M17 & M19 & M20 & M20 & P20 & P20 & EP20 & EP20 & EP20 \\
\hline \multirow{15}{*}{ Light } & 17 & & & & & & & & & & & & & & & & \\
\hline & 19 & & & & & & & & & & & & L7 & L7 & L7 & L7 & L7 \\
\hline & 21 & & & & & & & L7 & L7 & L8 & L8 & L8 & L8 & L8 & L8 & L8 & L9 \\
\hline & 23 & & & & & L7 & L7 & L8 & L8 & L10 & L10 & L10 & L10 & L10 & L10 & L10 & L10 \\
\hline & 25 & & & & L7 & L8 & L8 & L9 & L10 & L10 & L11 & L11 & L12 & L12 & L12 & L12 & L12 \\
\hline & 27 & & & L7 & L8 & L8 & L10 & L10 & L10 & L11 & L12 & L12 & L12 & M13 & M13 & M13 & M13 \\
\hline & 29 & & L7 & L7 & L8 & L10 & L10 & L11 & L11 & L12 & L12 & M13 & M13 & M14 & M15 & M15 & M16 \\
\hline & 31 & & L7 & L8 & L9 & L10 & L11 & L11 & L12 & L12 & M13 & M14 & M15 & M15 & M16 & M16 & M17 \\
\hline & 33 & & L7 & L8 & L10 & L10 & L11 & L12 & L12 & M13 & M14 & M15 & M16 & M16 & M17 & M18 & M18 \\
\hline & 35 & & L8 & L9 & L10 & L11 & L12 & L12 & M13 & M14 & M15 & M16 & M16 & M17 & M18 & M18 & M18 \\
\hline & 37 & L7 & L8 & L9 & L10 & L11 & L12 & M13 & M14 & M15 & M16 & M16 & M17 & M18 & M18 & M18 & M18 \\
\hline & 39 & L7 & L8 & L10 & L10 & L12 & L12 & M13 & M14 & M15 & M16 & M17 & M18 & M18 & M18 & M18 & M18 \\
\hline & 41 & L7 & L8 & L10 & L11 & L12 & M13 & M14 & M15 & M16 & M17 & M18 & M18 & M18 & M18 & P18 & P18 \\
\hline & 43 & L7 & L9 & L10 & L11 & L12 & M13 & M14 & M15 & M16 & M17 & M18 & M18 & M18 & P18 & P18 & P18 \\
\hline & 45 & L7 & L9 & L10 & L11 & L12 & M13 & M15 & M16 & M17 & M18 & M18 & M18 & P18 & P18 & P18 & P18 \\
\hline \multirow{15}{*}{ CEMIG } & 17 & & & & & & & & & & & & & & & & \\
\hline & 19 & & & & & & & & & & & & & & & & \\
\hline & 21 & & & & & & L9 & L9 & L9 & L9 & L9 & L9 & L9 & L9 & L9 & L9 & L9 \\
\hline & 23 & & & & & L9 & L10 & L10 & L10 & L10 & L10 & L10 & L10 & L10 & L10 & L10 & L10 \\
\hline & 25 & & & & L9 & L10 & L10 & L10 & L10 & L10 & L10 & L10 & L10 & & & M10 & M10 \\
\hline & 27 & & & L9 & L10 & L10 & L10 & M10 & M10 & M10 & M10 & M11 & M11 & M11 & M11 & M11 & M11 \\
\hline & 29 & & & L9 & L10 & L10 & M10 & M10 & M11 & M12 & M12 & M12 & M12 & M12 & M12 & M12 & M12 \\
\hline & 31 & & & L9 & L10 & M10 & M10 & M11 & M12 & M12 & M12 & M12 & M12 & M12 & P12 & P12 & $\mathrm{P} 12$ \\
\hline & 33 & & & L10 & L10 & M10 & M11 & M12 & M12 & M13 & P12 & P12 & P12 & P12 & P12 & $\mathrm{P} 12$ & $\mathrm{P} 12$ \\
\hline & 35 & & L9 & L10 & M10 & M11 & M12 & M12 & $\mathrm{P} 14$ & P14 & P14 & P14 & P14 & P14 & P14 & P14 & \\
\hline & 37 & & L9 & L10 & M10 & M13 & M14 & M14 & M15 & M15 & P14 & P14 & P14 & & & & \\
\hline & 39 & & L9 & L10 & M10 & M13 & M14 & M15 & M15 & M15 & P14 & & & & & & \\
\hline & 41 & & L9 & M10 & M11 & M13 & M14 & M15 & P15 & P15 & P15 & & & & & & \\
\hline & 43 & & L9 & M10 & P11 & M13 & M15 & P15 & P16 & P16 & P17 & P17 & & & & & \\
\hline & 45 & & L10 & M10 & P11 & M14 & M15 & P15 & P16 & P17 & P17 & P18 & P18 & & & & \\
\hline
\end{tabular}

${ }^{6} \mathrm{ELi}, \mathrm{Li}, \mathrm{Mi}$, Pi e EPi indicam postes tipos extraleve, leve, médio, pesado e extrapesado, respectivamente, com “i” metros de comprimento. Valores em negrito referem-se às dispersões das árvores-amostra. 


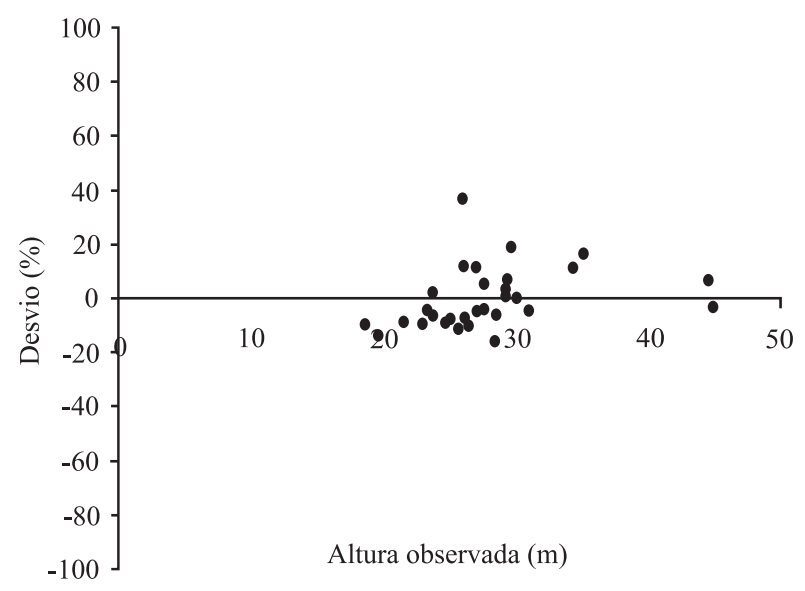

Figura 2-Distribuição dos desvios percentuais entre os valores observados e recuperados pela equação 4 utilizando os dados das 30 árvores-amostra.

Figure 2-Distribution of percentage deviations between values observed and recovered by equation 4 using data from 30 sample-trees.

\section{CONCLUSÕES}

Diante dos resultados, conclui-se que:

- A norma de classificação ABPM-E86 é mais eficiente do que as normas Light e CEMIG, no quesito melhor utilização da madeira.

-Autilização de modelos de afilamento (taper) para estimar diâmetros em diferentes alturas da árvore possibilita também quantificar mais de um tipo de poste por árvore.

- O método seguido neste estudo é indicado para realização de inventários florestais, com o objetivo de quantificação de tipos de postes de madeira, quaisquer que sejam as normas adotadas, independentemente do modelo de afilamento.

\section{REFERÊNCIAS}

\section{ASSOCIAÇÃOBRASILEIRADE PRESERVADORES DE MADEIRA - ABPM. Boletim de Preservação. Boletim Eletrônico}

Semanal, v.5, n.239, 2005. Disponível em: < http:// www.abpm.com.br/>. Acesso em: 3 maio de 2008.

AGÊNCIA NACIONAL DE ENERGIA ELÉTRICA ANEEL. Acompanhamento das Linhas de Transmissão. Disponível em: <http:// www.aneel.gov.br/area.cfm?idArea $=38>$ Acesso em: 26 abr. de 2008.

Revista Árvore, Viçosa-MG, v.35, n.4., p.875-882, 2011
ANTUNES, F. Z. Caracterização climática do Estado de Minas Gerais: climatologia agrícola. Informe Agropecuário, v.12, n. 138, p.9-13, 1986.

BENTO, F. R. et al. Estudo da durabilidade de postes de madeira preservados com CCA por meio do controle da retenção de As, Cu e Cr. In: CONGRESSO BRASILEIRO DE ENGENHARIA E CIÊNCIA DOS MATERIAIS, 15., 2002, Natal. Anais...Natal: 2002.

CAMPOS, J. C. C.; LEITE, H. G.; SOUSA, R. N. Classificação de árvores de eucalipto para postes. Boletim Técnico SIF, p.10-19, 1991.

DEMAERSCHALK, J. P. Converting volume equations to compatible taper equations. Forest Science, v.18, n.3, p.241-245, 1972b.

GARAY, L. Tropical forest utilization system. VIII. A taper model for entire stem profile including buttressing. Seatlhe: Institute of Forest

Resources, College of Forest Resources, University of Washington 1979. 64p. (Contrib. 36).

LEITE, H. G. et al. Função de afilamento para Virola surinamensis (Roll.) Warb. Revista Árvore, v.30, n.1, p.99-106, 2006.

LIMA, F. S. Análise de Funções de “Taper” Destinadas á Avaliação de Multiprodutos de Árvores de Pinus elliottii. 1986. 79f. Dissertação (Mestrado em Ciência Florestal) - Universidade Federal de Viçosa, Viçosa, MG, 1986.

MACDICKEN, K. G.; VERGARA, N. T.

Agroforestry: classification and management. New York: John Wiley \& Sons, 1990. 382p.

REMADE. Revista da Madeira. A madeira de eucalipto para postes. Disponível em: < http:// www.remade.com.br/br/revistadamadeira_materia.php? num $=409 \&$ subject $=$ Postes \& title $=$ A\%20madeira\%20de \%20eucalipto\%20para\%20postes >. Acesso em : 25 abr. de 2008.

VIEIRA, A. R. R.; FEISTAUER, D.; SILVA, V. P. Adaptação de espécies arbóreas nativas em um sistema agrossilvicultural, submetidas a extremos climáticos de geada na região de Florianópolis. Revista Árvore, v.27, n.5, p.627-634, 2003. 